\title{
Research on Cloud Computing-based Open Virtual Computer Lab Liling Zhang
}

Dept of Computer Science and Engineering, Hubei University of Education, Wuhan, China,430205 hu_tu@qq.com

\begin{abstract}
In order to solve the problem of low utilization rate of resources existing in physics experiment teaching, this paper proposes an open virtual laboratory based on Cloud Computing (VCL) design scheme, with the scheme, the administrator can provide services based on the on-demand/reserved for students and teachers in any time and any place.
\end{abstract}

Keywords: cloud computing, virtual computer lab.

\section{基于云计算的开放式虚拟计算实验室（VCL）研究}

\author{
章丽玲 \\ 湖北第二师范学院计算机科学与工程系, 武汉, 中国, 430205 \\ hu_tu@qq.com
}

\begin{abstract}
摘要: 为解决现有物理实验教学资源利用率 低的问题，本文提出了一种基于云计算的开 放式虚拟实验室（VCL）的设计方案，该方 案可以使管理员能够在任何时间任何地点通 过按需提供/预约的方式为所有学生和教师 提供实验所需的服务环境。
\end{abstract}

关键词：云计算、VCL

\section{一、引言}

目前，普通高校的实验室非常多，而且 相互独立, 就来我们湖北第二师范学院来说, 各个院系都有自己独立的实验室, 物电学院 有物电方面的专业实验室, 计算机学院有计 算机专业的专业实验室, 就是同一个学院, 由于专业方向不同，所设的实验室也不同， 如计算机学院有网络实验室、嵌入式实验室、 网络安全实验室、综合布线实验室、软件实 验室、多媒体实验室等等。由于这些实验室 仅仅针对某一个具体的专业, 实验设备经常 闲置, 资源利用率非常低, 而另一方面, 专 业实验室往往也不能满足学生综合实验的需 求, 对于一些较复杂的科研实验要求, 则需 要引进昂贵的设备。为此, 如何很好地利用 现有的实验室资源, 让它更好地为教学和科 研服务, 成为摆在实验室管理人员和决策者
面前的一个难题。

为进一步解决现有物理实验室教学在时 空、管理、教学资源利用等方面的难题, 本 文给出了基于云计算的开放式虚拟计算实验 室 (VCL) 的设计方案, 以实现实验室教学 资源在教学和科研的充分利用, 利用此方案, 管理员能够在任何时间任何地点通过按需提 供预约的方式为所有学生和教师提供实验 所需的服务环境。

\section{二、云计算概述}

\section{1 云计算简介}

所谓的云计算可以被看成是网格计算和 虚拟化技术的融合, 即利用网格分布式计算 处理的能力, 将 IT 资源构筑成一个资源池, 再加上成熟的服务器虚拟化, 存储虚拟化技 术，以便用户可以实时地监控和调配资源。 云计算的核心思想是将大量用网络连接的计 算资源统一管理和调度, 构成一个计算资源 池向用户提供所需的服务。云计算旨在通过 互联网把多个成本相对较低的计算实体整合 成一个具有强大计算能力的完美系统, 并且 借助 SaaS 、 PaaS 、 IaaS 、 MSP 等先 进的商业模式把这强大的计算能力分布到终 
端用户手中。云计算技术的体系结构图如图 (1) 所示。

服务接口 服务注册 服务查找 服务访问 服务工作流

图 1 云计算技术的体系结构图

\section{2 云计算对高校实验教学的影响}

正如前面所说，假如我们将云计算技术 运用到实验教学当中, 能将实验教学资源也 像水电煤气这些公共设施一样给师生使用, 这对于高校实验教学来说, 无疑是一个重大 的突破。

首先，针对高校信息化建设成本高的问题， 将云计算理念运用到实验室建设方面, 可以 大大减少对实验教学投入的硬件成本, 可将 实验教学资源得到合理的充分利用, 避免对 实验室的重复建设和减少对实验设备使用过 程中的损耗。其次，云计算的使用，可以将 实验室的实验资源进行智能管理, 各系部的 教师或者学生根据自己需求, 提供给他们一 种弹性扩展的实验平台, 提高了实验教学管 理的效率。更为重要的是, 云计算能够为教 育信息化建设提供技术支撑和交付方式，构 建不同的云服务, 共享这些丰富的实验教学 资源, 让师生之间的教与学能够充分融合在 一起, 并且可以随时随地地进行沟通、交流, 增加教与学的互动性, 激发学生的兴趣、想 象力和创造力, 充分调动学生学习的积极主 动性和追求真知实干的严谨态度, 真正做到 实验教学所要求 “求真、创新” 的目的。利 用云计算通过共享开发测试资源和远程桌面 共享的方式, 可以实现 “每个师生都有一个 属于自己实验室——虚拟实验室”的设想。

\section{三、基于云计算的开放式虚拟计算实验室}

\section{（VCL）的研究}

\section{1 基于云计算的开放式虚拟计算实验室架 构}

基于云计算的开放式虚拟计算实验室架 构图如图 2 所示:

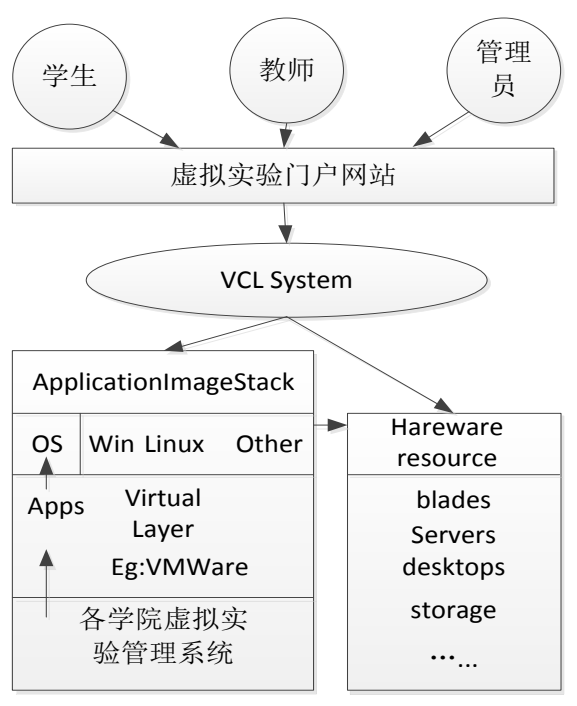

图 2: 基于云计算的开放式虚拟计算实验室架构图

\section{2 虚拟计算实验室 (VCL) 组成}

VCL 虚拟计算实验室的目的是设计和 配置以一种经济、成本有效的方式为以教育 和研究为导向的大学提供服务的云计算系统, 提高实验室资源的利用率。从图 2 可知, VCL 虚拟计算实验室架构包括以下组要组件。

1、虚拟实验门户网站, 即终端用户访问界 面（基于 Web）。

2、 VCL 管理器（整个架构的核心）

3、ApplicationImageStake 映像库

4、计算、存储和各种网络硬件资源

\subsection{1 虚拟实验门户网站}

虚拟实验门户网站作用之一是方便学生、 教师和管理员预约实验所需资源, 对资源进 行全局统一的分配, 可以充分提高计算机的 硬件与软件资源的使用效率与充分发挥计算 
机系统的性能。作用之二是：通过虚拟实验 门户网站, 可以向学生、教师、管理员提供 实验教学管理功能。教师既可搭建典型实验 或调取实验案例, 方便地向学生布置实验任 务, 还可在实验结束后查看学生的实验结果, 给出实验成绩和评价。学生可将实验中的经 验、教训、收获和问题在论坛上发布, 教师 可将实验中的不足提出来，师生共同探讨。 教师从中可以得到及时的实验教学反馈信息, 以便调整实验教学的进度和深度。学生也可 从中吸收别人的经验, 快速提高自己的能力。

\subsubsection{VCL 管理器}

VCL 管理器最重要的功能是对各种资源 进行统一管理, 这些资源包括各种硬件资源、 操作系统资源、开发平台资源、数据库资源、 应用软件资源等。VCL 利用 Application Image Stack 来实现资源的映像, VCL 可 以是面向裸机的映像, 也可以是基于

VMware 的映像。VCL 系统接收 Web 界面 的用户请求并处理请求, 如果无法找到已经 加载了所需映像的真实的或虚拟的服务器, 那么它将选择任何满足此映像所需规范的服 务器, 动态加载所需的映像。如果所有服务 器处于繁忙状态, 那么Web 界面将通知学 生在可用的时段使用系统。

VCL 管理器的第二个功能是实现资源预 约服务, 根据所请求的环境类型 一 不管 是裸机映像、实验室机器或虚拟机映像, 资 源预约服务都将确保映像被加载并能够用于 请求。资源预约服务器主要负责资源使用日 程安排和资源管理。基于用户的请求，根据 管理节点反馈的资源信息选择最优的调度方 案。它实现了用户 / 用户组权限分配、用户 / 用户组权限检测、资源的预约、资源及其 镜象管理(资源及镜象的增删改、资源分组、 版本管理等)以及跟管理节点间的通信(主要 是镜象资源的可用状态、使用情况等信息)， 还实现了资源预约的汇总统计等功能。相应 地, 预约数据库除了包含物理资源的镜象描 述信息及用户对镜象信息的预约情况等外， 还包含了用户信息、用户分组、用户权限和
资源访问权限等权限信息, 当然还保存了相 关的系统使用日志信息。

VCL 管理器第三个功能是提供一个开源 的Web 服务器, 该Web 服务器提供了请求、 管理和治理所有 VCL 资源的工具。Web 界 面支持经过身份验证的用户，显示他们有权 使用的应用程序列表, 并允许他们预定对某 个应用程序的使用，可以立即使用这个应用 程序，或者在未来指定的一段时间内使用。 未来时间的范围和预定的长度可以进行定制, 并可以因客户而异。Web 界面提供的主要工 具包括:

映像创建 - 该界面允许用户创建定制 的环境。

映像版本控制 - 这个界面允许特权用 户创建同一映像的多个版本。

管理用户 - 为用户提供了特权控制, 它 通过 Web 界面向用户授予不同程度的控制 权。

管理资源 - 该界面提供一种方法来调 度资源池中的资源。

\subsection{3 映像库 (ApplicationImageStack)}

映像库是一种典型的软件栈结构, 包含 底层的操作系统, 运行在操作系统之上的中 间层, 可以想象成虚拟映像层, 最上一层是 应用软件层, 对应于各学院的虚拟实验管理 系统, 二层的虚拟映像可以被加载到裸机， 或加载到所选的操作系统/应用程序虚拟环 境。如果用户所需的映像组合不可用, 用户 有权从 VCL 组件库中构建自己的映像。

\subsection{4 计算、存储和各种网络硬件资源}

学校的所有硬件资源, 包括网络设备、 服务器、各种存储设备、安全设备、通信设 备等等。VCL 管理器提供了一个资源池, 可 以供用户使用。资源的分配以将要执行计算 的特定应用程序为依据。存储和网络资源是 动态的, 用于满足不同用户需求。 


\section{四、总结}

基于云计算的开放式虚拟计算实验室能 够促进学生学用结合, 实验的安排更加灵活 方便且不受时间空间限制。只要有网络的地 方就可以动手做实验, 实现真正意义上的开 放实验室。虚拟实验可减少实验设备的维护 强度, 缓解当前实验设备不足, 实现理论教 学和实践教学的有机融合, 可以解决计算机、 电子、通信等学科中的实验教学问题, 完善 现有实验教学体系。以实现实验室教学资源 在教学和科研的充分利用, 用户能够在任何 时间任何地点通过按需提供/预约的方式为 各种用户提供实验所需的服务环境。

\section{References}

[1] GUI Guan-xun.Design and Implementation of a Computer Experimental Teaching
Platform Based on Cloud Computer. Research and Exploration in Laboratory. Vol 32 No.10 Oct.2013

[2] Justin Cappos, Ivan Beschastnikh, Arvind Krishnamurthy,et al.Seattle:A platform for education cloud computing[J].Proceedings of the 40th ACM technical symposium,2009(3):111-115

[3] Marianne C.Murphy, Marty McClelland, Computer Lab to Go:A "Clou"Computing Implementation[J].Proc ISECON 2008,2008(11):1-10

[4] Justin Cappos,Ivan Beschastnikh,Arvind Krishnamurthy, et al.Seattle:A platform for educational cloud computing $[\mathrm{J}]$.Proceedings of the 40th ACM technical symposium,2009(3):111-115 